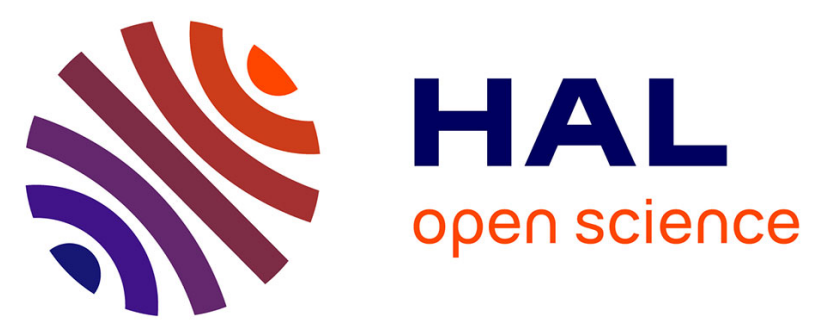

\title{
Computer-Aided Collaborative Work into War Rooms: A New Approach of Collaboration
}

Jeremy Ringard, Samuel Degrande, Stéphane Louis-Dit-Picard, Christophe Chaillou

\section{- To cite this version:}

Jeremy Ringard, Samuel Degrande, Stéphane Louis-Dit-Picard, Christophe Chaillou. Computer-Aided Collaborative Work into War Rooms: A New Approach of Collaboration. Human Interface and the Management of Information. Designing Information Environments, pp.835-844, 2009, Human Interface 2009: Human Interface and the Management of Information. Information and Interaction, 10.1007/978-3-642-02559-4_90. hal-02492198

\section{HAL Id: hal-02492198 \\ https://hal.science/hal-02492198}

Submitted on 27 Feb 2020

HAL is a multi-disciplinary open access archive for the deposit and dissemination of scientific research documents, whether they are published or not. The documents may come from teaching and research institutions in France or abroad, or from public or private research centers.
L'archive ouverte pluridisciplinaire $\mathbf{H A L}$, est destinée au dépôt et à la diffusion de documents scientifiques de niveau recherche, publiés ou non, émanant des établissements d'enseignement et de recherche français ou étrangers, des laboratoires publics ou privés. 


\title{
Computer-Aided Collaborative Work into War Rooms: A New Approach of Collaboration
}

\author{
Jeremy Ringard, Samuel Degrande, Stéphane Louis-dit-Picard, \\ and Christophe Chaillou \\ ALCOVE, bâtiment IRCICA \\ Parc scientifique de la Haute-borne, 50 av. Halley \\ 59650, Villeneuve d'Ascq, France \\ \{Jeremy.Ringard, Samuel.Degrande, Christophe.Chaillou\} @lifl.fr, \\ stephane.louisditpicardeorange-ftgroup.com
}

\begin{abstract}
This paper presents the realization of a new software and hardware platform for collocated collaborative work. Our objective is to take the most of the various competences of the teammates. We have created an architecture named MVT (model, view, tool) for supporting collaborative interaction in warroom-like environments. This software distribution offers various interactions modalities, allowing multi-skilled teams to collaborate using different input devices, thanks to multiple visualization and interaction channels.
\end{abstract}

Keywords: collocation, collaboration, CVE, war room, teamwork.

\section{Introduction}

For several years, technologies that could allow efficient real-time collaboration between co-workers separated by a geographic distance have been a major problematic for scientific community. Increasing needs from industry regarding project management brought researchers about working on the development of some tools allowing several teammates to work together on a common project. Several approaches have been issued, through technologies like videoconferencing, document sharing applications, or collaborative virtual environments (CVE). CVEs represent a major subject of research: it consists in bringing several distant users together in a common $2 \mathrm{D}$ or $3 \mathrm{D}$ environment.

These CSCW (computer supported collaborative work) technologies have mainly focused on distant collaboration, which is a situation that occurs while working on coconception activities, but some real collaboration's use cases are still left aside: in a real situation, the co-workers are more likely to be located in the same building. Moreover, when the project requires the intervention of distant users, there is a poor probability for the entire team to be geographically dispersed. This involves group to group collaboration, rather than peer to peer. Consequently, companies are massively adopting others collaboration strategies for teamwork: the "war room" configuration [1][2].

The main advantage of this type of collocated work resides in the permanent and direct contact between the co-workers which allow them to be able to respond immediately to any unexpected issues. 
Despite the massive adoption of this type of configuration by companies, very few scientific research have focused on software support for collocated work such as war rooms, let-alone regarding interaction on virtual models.

In this study, we present a new approach of computer aided collaborative work, through the development of a software that can be assimilated to a traditional CVE, but optimized for collocated multimodal collaboration. Our proposition is a new software and hardware platform that allows a team of users to interact and communicate in the same way than in a war room-like environment. The software platform is especially designed for scenarios like working on virtual models (from architecture to complex mechanic systems). Every user is able to interact differently on the objects thanks to a large range of tools.

Before describing precisely our work, we will firstly present the state of art about collaborative software solutions. The next section will describe the concept and the problems addressed by our war room, before detailing the functionality of our prototype. We will conclude this paper by presenting the future works that are planned.

\section{State of Art}

Among all the technologies developed to assist teamwork on a project by synchronous interaction, tools like CVEs are the most complete. This kind of support consist in allowing people to cooperate by virtually bringing them together in a virtual environment (most likely 3D), providing them several tools for interacting on shared virtual objects, and some distant communication means[3][4].

The major benefit brought by CVEs is the possibility to set up purely virtual teams. However, those technologies turn out to be not properly adapted to some use cases: in most of the real situations, the working group is composed at least partly by some collocated people.

For these reasons, the working methods have been adapted to this parameter, through the use of war rooms. Jason Leigh has published some complete works regarding this kind of configuration [5]: Working in a war room consists in bringing a whole collaborators team in a closed space, for working on a common project. This project can be a simple brainstorming task, crisis management, or every type of task that requires good reactivity and instant feedbacks between co-workers.

Regarding material, the observation of a "traditional" war room is quite interesting, because of the diversity of writing surfaces that are involved in the room [5]. We can cite, among other things, a surface dedicated to the permanent display of some particular information (a corkboard to pin the planning). In the same way, another large scale surface can be used for several teammates to work together on one subtask (a blackboard). Each surface is parented to one kind of subtask.

An interesting fact regarding this surfaces diversity is the spatiality of processed data: Leigh et al. [5] highlights the fact that people talking about one element of the project naturally refers to the place where the associated surface is located. Generally, the link between spatial distribution of data and the sequence of collaboration is strong. Mark et al. [1] mention the fact that every user establishes a interdependencies map, that allow them to immediately identify which teammate is 
the most likely to perform a subtask, depending of everyone's competences. The efficiency of a collocated team have been highlighted in multiple papers [6][2][7].

In our vision of the war room, we can associate this notion of spatial and human distribution of the competences with the multiplicity of writing surfaces: We propose to enrich these surfaces by replacing them with more complex interactive devices. As a consequence, a direct link can be established between the devices and the users' competences.

The major consideration enhancing the war room efficiency is a consequence of the natural human contact: Direct communication, without using third-party technologies such as videoconferencing or virtual avatars, improves comprehension thanks to non-verbal communication items that are not (or not properly) available when working through a virtual environment. Moreover, the spatial proximity of the teammates provides a better accessibility, and a favorable atmosphere for teamwork. Finally, the mutual comprehension between each user is enhanced by informal conversations that could occur between people in the room [8].

Few approaches tackle the notion of collocated collaboration, especially when using multitouch tabletop devices [9][10]. Unfortunately, those researches only offer one single interaction modality, by working exclusively on tabletop devices. Streitz et al. have developed "I-LAND", a collocated, multi-computer platform for collaboration [11]. However, this solution does not provide multiple heterogeneous displays and tools for interacting on the documents.

Some other papers have been presented, describing collaboration between a group and a single distant user, or more generally group to group collaboration [12][13][14]. However, those approaches don't provide any real interaction activity, and rather focus on communication tasks.

\section{Computer Aided War Room: The Concept}

Even if the papers described above bring some interesting facts for setting up collocated collaboration, none provide a generic tool for virtual interaction in a war room-like environment. This is precisely what we are developing, through the realization of a computer support adaptable to several scenarios. Therefore, our proposition consists in a single room equipped with various workstations, and a virtual environment linked to each computer, allowing collaborative interaction.

Our interpretation is different from standard CVEs, since the notion of software support is not used in the same way (fig. 2):

- According to CVEs, the couple user-computer is fixed, so this couple is considered as one single entity. As a consequence, in a way, the collaboration do not occurs between users, but between computers, The PC to PC link offering a communication channel (videoconferencing, avatars, telepointers,...), and an interaction broadcasting channel.

CVEs are not adapted for collocated work: grouping several CVE-connected computers in the same room do not fit our purpose, because most CVE applications provide a WYSIWIS ("What you see is what I see"), relaxed or not. Therefore, the available interactions are the same for each user. This concept does not fit the fact that each user is specialized for one kind of task. Moreover, the fact that each user 
is permanently linked to his workstation lower their freedom, as well as the cerebral stimulation provided by collocated teamwork.

- Contrary to the configuration discussed above, our approach emphasizes diversity, from visual representations and from interaction.

In our proposition, the major collaborative entity is composed of the whole team. The semantic links established between the users and their workstations are breakable and modifiable as required, depending of the needs of each teammate at every instant. The stations count is independent from the physical team. The interaction objects, as well as the virtual tools, can be diffused freely among the workstations (including simultaneous displays) depending of the desired activity distribution.

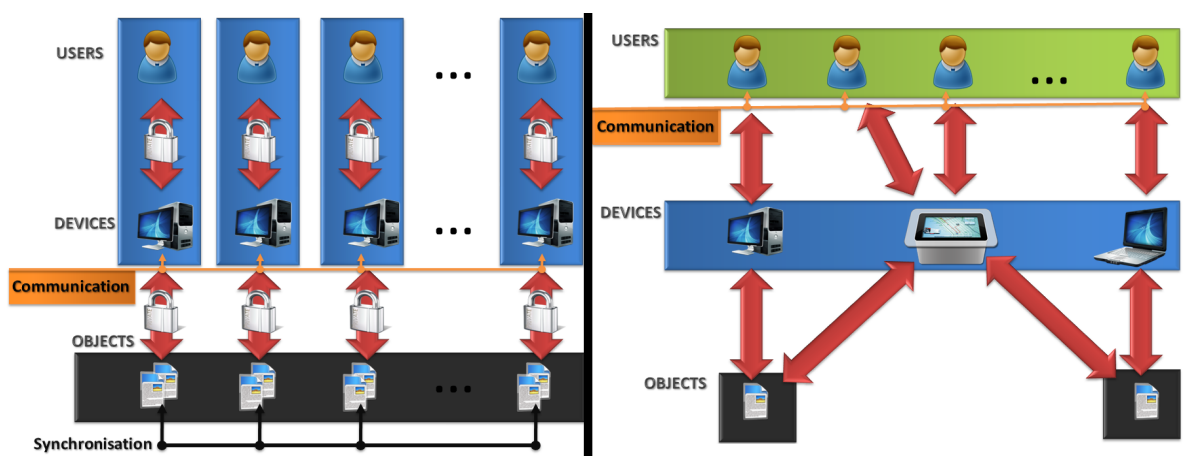

Fig. 1. Left: Traditional CVE concept. Right: war room application on the right

The development of our war room essentially relies on three elements: Firstly, the heterogeneity of the hardware devices provided to the users, then the interfaces plasticity, and finally the spatial localization of the teammates in the room.

\subsection{Hardware Heterogeneity}

The devices catalog available now allows us to benefit from a large variety of interaction modalities and visualization surfaces. It appears natural to take advantage of this diversity by providing on each workstation the most adapted interaction tools and display methods. When collaborating on a large scale project, multiple different skilled professionals are likely to be involved. As a result the users' needs regarding the project's object are not the same, depending of the human specialties involved.

Let us take a simple example: A vehicle conception project. This kind of task leads to the presence of several people: A specialist of electronic, who would be in charge of the electric cables repartition inside the vehicle's body, will be more likely to work with detailed blueprints illustrating the inside of the vehicle. In the same time, another specialist (a designer for instance) working on the same project need to work directly on the shape of the car body. This subtask may be easier to perform by interacting directly on a $3 \mathrm{D}$ view. 
Consequently, even when working on the same project, the collaborators' visions are singularly different. Our choice is to take advantage of the hardware diversity to answer those different needs. A multitouch tabletop display, for instance, will be more likely to be used for 2D interaction. In the same way, a large scale stereoscopic screen coupled with a 6 dof input device will appear perfectly adapted for interaction in a 3D environment. Some mobile terminals like PDAs or smartphones, can be useful too, providing tools adapted to the mobility of these devices.

\subsection{Plasticity}

To extend the use of hardware diversity in the war room, we want to propose a functionality that can be assimilated to the notion of interface plasticity [15]. While working in the war room, each change in interaction modality potentially involve a move from the user, to pass from one computer to another. However, in the case of a short-time interaction, it could be useful to provide to the user the ability to import dynamically the tools and/or the objects necessary for this interaction, avoiding physical move in the room. This proposition involves the necessity to adapt dynamically the interface and the interaction modalities from the second workstation to a new display/input device that was not originally planned. We notice that the two notions of hardware heterogeneity and of spatial distribution of activity are closely linked.

\subsection{Collocation}

This third subsection is focused on a specific goal: To take advantage of collocation and the associated phenomena for enhancing collaboration. We have to make sure that the spatial repartition (of data and users), and software tools are pertinent enough to provide optimum conditions. The common denominator between the multiple questions is the users' behavior during collaboration. For instance, regarding nonverbal communication, the fact that the users are brought together in the same room allow them to naturally benefit from the gesture, the gaze direction, or facial expressions. All these elements have a semantic signification that allows the teammates to better understand each other. This could significantly enhance the collaboration, especially in our war room, where virtual and real environment coexist to constitute a single mixed environment. Deictic gesture, for instance, which is used to show something to the others, not only apply to the $3 \mathrm{D}$ scene displayed, but can refers to the physical space, i.e. the room.

Moreover, we have seen in the section above that co-workers established several dependences map, including tasks, specialties, and data's localization. This phenomenon may be determining regarding the collaboration's efficiency. Consequently, the spatial distribution of the physical devices could influence the teamwork. In the same way, the distribution of virtual objects across the workstations must keep coherence regarding the dependences map.

An evaluation should be performed to understand exactly, through several different configurations, what choices are the most judicious, and what the potential effects of the distribution on teamwork are. 


\section{War-Room Software Support Proposal}

\subsection{Software Design}

We have chosen to start from a existing platform to develop the war room software: SPINI3D [3]. SPINI3D is a CVE software platform whose major particularity is the fact that every object in the environment is composed of a structure close to the well known Model-View-Controller structure (MVC), named Model-View-Tool (MVT). (fig. 2).

In the MVT architecture, the tool is a component that is used to interact on the virtual object by modifying the data associated to this object. The data describing an interaction object is stored in a dedicated component: the "model". The third component, the "view", is a graphical representation of this object, for display. The view is a form of translation of the model's data. A single object can have several views.

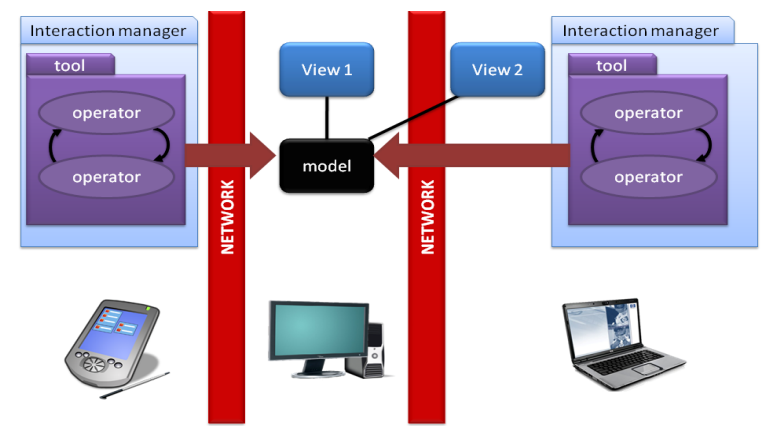

Fig. 2. An example of distribution of the application

Contrary to the standard SPINI3D architecture, the presence of every virtual object on the devices is not always necessary. This is why we have developed a completely free distribution of these components between the workstations, by adding a network communication layer on the links between the Model, the View, and the Tool components. So the three components can be distributed on three different computers acting as a single MVT structure, the distribution being transparent to the platform's high-level layers.

In practical terms, this enables the fact to split the tasks and to make them independent from the hardware configuration of the war room. Various scenarios are possible (fig.2), such as:

- In a configuration providing one device optimized for 3D interaction, while another station offers a better 3D display, the user can use the two computers simultaneously: the first one with the input device (like a remote control), and the second one for visualization.

- If a user is working using a 3D view, he is able to send (or duplicate) his local view to a large scale display workstation, to make the object viewable by the teammates.

This distributed architecture enables multiple possibilities that we have illustrated through the realization of a prototype, presented in the next section. 


\subsection{The Prototype}

To illustrate the various features provided by our architecture, we have developed a prototype, including 3 different computers (fig.3):

- A 3D accelerated PC, connected to a large scale projection display. This station is also equipped with a wireless interaction stylus (prototype input device [16]) that allows 3D manipulation, and a ARTrack infrared tracking system.

- An interactive tabletop, dedicated to 2D interaction through a tactile interface.

- A standard laptop.

The scenario proposed is a car review application, allowing users to perform simple interactions on a virtual vehicle. On the tabletop, a $2 \mathrm{D}$ interface is available for interactions on blueprints views. Thanks to this interface, the user is able to select a color value and to drag it to the car parts. In the same way, the doors can be manipulated with sliders or buttons, which fit better on a 2D interface. Naturally, every interaction performed on this $2 \mathrm{D}$ interface is directly displayed on the $3 \mathrm{D}$ views. The others workstations are used to provide direct interaction on the car's 3D model, through different parts manipulation.

The 3D view is displayed on one station at a time (laptop or 3D optimized PC). Thus, the users are able to "teleport" the car's 3D view from one workstation to the other, depending of the computer they want to use for visualization and interaction. As those two computers don't provide the same computing power, the level of detail of the 3D mesh used as a view is different, depending on the displaying station. Moreover, to avoid too many moves from the teammates in the room, they are able to teleport their pointer between the computers, allowing them to interact at a distance from the display station, and using their local input device for interaction. Consequently, the coworkers are able to interact simultaneously on the 3D car model, using a common display surface, but using their own input devices, and so different interaction modalities.

This demonstrator has been presented during the professional exhibition "Laval Virtual 2008" in France, and has received a very positive feedback from the users. A lot

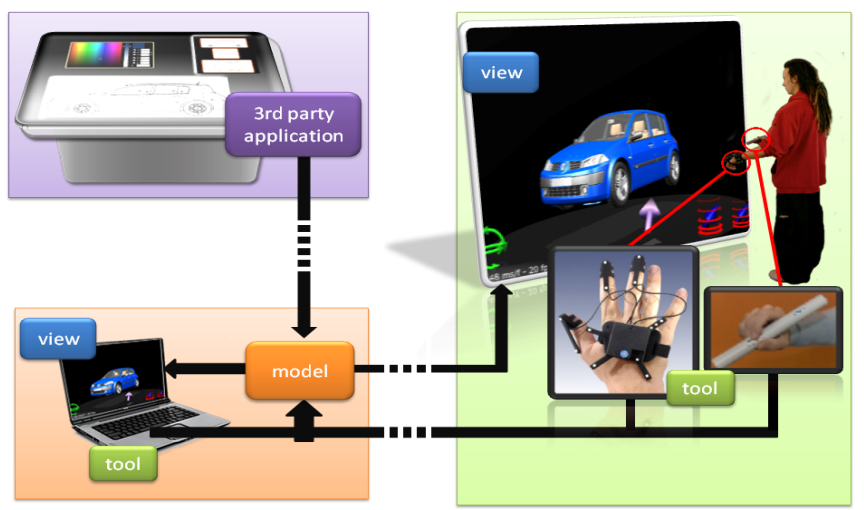

Fig. 3. The prototype 
of visitors have shown a great interest in our activity repartition, especially about the complementarity of each viewing/input device. After a brief presentation of the concept and of the presence of a single distributed object, the use of the installation has not presented any major issue. The ability to modify a $3 \mathrm{D}$ object thanks to the $2 \mathrm{D}$ interface has been pointed as interesting for the most part of the users. A lot of them have expressed their desire to enable distant collaboration with the room.

\section{Future Works}

\subsection{Mixed Presence}

While working on large scale projects, bringing every teammate in the same geographic point is not always possible. Therefore, it can be interesting to keep all the advantages provided by CVEs by allowing a third party user (or team) to integrate the collocated team without moving.

The major issue raised by this feature lies in an asymmetry, which is involved in two ways: First, human asymmetry, already known as "mixed presence" [17]. Groups involved in distant collaboration are confronted to a problematic communication configuration.

Secondly, hardware asymmetry can be problematic. If a distant user wants to join the team, he must be able to get connected to the war room from his personal computer, without suffering the lower interaction possibilities of his devices.

These two asymmetries must be evaluated on our prototype to understand how to avoid communication issues between distant co-workers and how to enhance collaboration in such a mixed environment.

\subsection{Semantic Data}

To formalize the technology presented above, we can say that the software side of the war room focuses on multiple "interaction channels". A channel is defined by a display type (2D, 3D, video...), and its associated interaction modalities. Even if it is possible to factorize several interactions between channels to keep them compatibles with any interface, some other interactions may be only available for one channel, without any equivalence on the others.

In a situation presenting a single object displayed on two workstations through different channels, every user must be aware of what the others are doing: To maintain the "social awareness", the interactions (and ideally, the whole interaction sequence) must be displayed on every station, even if the channel are not compatibles.

The solution we are currently working on consists in changing the semantic level of the interactions: In a improved-MVT structure as described above, most of the data stored in the model are geometric or visual data. By raising the level of this data to use real semantic information, the graphical interpretations can adapt to the corresponding display channel.

The next step in this work is to create a precise architecture introducing semantic abstraction of the MVT model: this solution requires the introduction of software adapters to convert the semantic data into geometric one. In this way, the structure would approach some known models such as PAC (presentation, abstraction, control), 
that can be used for semantic abstraction of data. The main difference between improved-MVT and these models lies in the absence of a generic "tool"-like component in PAC.

\section{Conclusion}

The proposition we have presented in this paper allows multiple users to collaborate in a collocated environment, while taking the most of the available hardware diversity. The distribution of MVT components between the workstations of the war room provides to the users a great flexibility, allowing them to be fully independent from their position in space, from the display surfaces, and from the input devices. This independence allows taking into account the teammates' specialties by providing them the most adapted interactions modalities and display methods for the subtask they want to perform. Moreover, this solution offers very good inter-users communication, thanks to the direct contacts allowed by collocation.

The spatial repartition of the activity, as well as the possibility to invite any distant user to virtually join the collocated team, raises some questions regarding the ability to collaborate in such an environment. As a consequence, an evaluation has to be performed to answer these questions. In the same way, developing the ability to set up a group to group collaboration using war rooms, taking into account hardware and human heterogeneity constraints, represents a new question.

Acknowledgments. We would like to thank Dominique Pavy and Arnaud Bouget from Orange Labs for their participation to realize the prototype. This work is funded by the ANR Part@ge project (06 TLOG 031) and by the IRCICA research federation from CNRS.

\section{References}

1. Mark, G.: Extreme collaboration. Commun. ACM 45(6), 89-93 (2002)

2. Teasley, S., Covi, L., Krishnan, M.S., Olson, J.S.: How does radical collocation help a team succeed? In: CSCW 2000: Proceedings of the 2000 ACM conference on Computer supported cooperative work, pp. 339-346. ACM, New York (2000)

3. Picard, S.L.D., Degrande, S., Gransart, C.: A corba based platform as communication support for synchronous collaborative virtual environment. In: M3W: Proceedings of the 2001 international workshop on Multimedia middleware, pp. 56-59. ACM, New York (2001)

4. Margery, D., Arnaldi, B., Chauffaut, A., Donikian, S., Duval, T.: Multi-threaded or modular animation and simulation kernel or kit: a general introduction. In: Virtual Reality International Conference, pp. 101-110 (2002)

5. Leigh, J., Johnson, A., Park, K., Singh, R., Chowdhry, V.: Amplified collaboration environments. In: VizGrid Symposium (2002)

6. Olson, J.S., Covi, L., Rocco, E., Miller, W.J., Allie, P.: A room of your own: what would it take to help remote groups work as well as collocated groups? In: CHI 1998: CHI 98 conference summary on Human factors in computing systems, pp. 279-280. ACM, New York (1998) 
7. Teasley, S.D., Covi, L.A., Krishnan, M.S., Olson, J.S.: Rapid software development through team collocation. IEEE Trans. Softw. Eng. 28(7), 671-683 (2002)

8. Bos, N., Olson, J., Nan, N., Shami, N.S., Hoch, S., Johnston, E.: Collocation bindness in partially distributed groups: is there a downside to being collocated? In: CHI 2006: Proceedings of the SIGCHI conference on Human Factors in computing systems, pp. 1313-1321. ACM, New York (2006)

9. Nacenta, M.A., Pinelle, D., Stuckel, D., Gutwin, C.: The effects of interaction technique on coordination in tabletop groupware. In: GI 2007: Proceedings of Graphics Interface 2007, pp. 191-198. ACM, New York (2007)

10. Tang, A., Tory, M., Po, B., Neumann, P., Carpendale, S.: Collaborative coupling over tabletop displays. In: CHI 2006: Proceedings of the SIGCHI conference on Human Factors in computing systems, pp. 1181-1190. ACM, New York (2006)

11. Streitz, N.A., Geißler, J., Holmer, T., Konomi, S., Müller-Tomfelde, C., Reischl, W., Rexroth, P., Seitz, P., Steinmetz, R.: 1999. i-LAND: an interactive landscape for creativity and innovation. In: Proceedings of the SIGCHI Conference on Human Factors in Computing Systems: the CHI Is the Limit, Pittsburgh, Pennsylvania, United States, May 15 - 20, 1999. ACM, New York (1999)

12. Bezerianos, A., McEwan, G.: Presence disparity in mixed presence collaboration. In: CHI 2008: CHI 2008 extended abstracts on Human factors in computing systems, pp. 32853290. ACM, New York (2008)

13. Mcewan, G., Rittenbruch, M., Mansfield, T.: Understanding awareness in mixed presence collaboration. In: OZCHI 2007, pp. 171-174. ACM, New York (2007)

14. Mark, G., Abrams, S., Nassif, N.: Group-to-group distance collaboration: examining the "space between". In: ECSCW 2003: Proceedings of the eighth conference on European Conference on Computer Supported Cooperative Work, Norwell, MA, USA, pp. 99-118. Kluwer Academic Publishers, Dordrecht (2003)

15. Demeure, A., Calvary, G.: Plasticity of user interfaces: towards an evolution model based on conceptual graphs. In: IHM 2003, pp. 80-87. ACM, New York (2003)

16. Ecole Supérieure d'Informatique, Electronique, Automatique. ESIEA, http: / / www.esiea.fr

17. Tang, A., Boyle, M., Greenberg, S.: Display and presence disparity in mixed presence groupware. In: AUIC 2004: Proceedings of the fifth conference on Australasian user interface, Darlinghurst, Australia, pp. 73-82. Australian Computer Society, Inc. (2004) 